\title{
Efficient Cloud Data Hosting Availability
}

\author{
P S V S Sridhar1), Ronnie Caytiles2)
}

\begin{abstract}
:
More and additional enterprises and organizations square measure hosting their knowledge into the cloud, so as to cut back the IT maintenance price and enhance the info responsibility. However, facing the various cloud vendors further as their heterogenous valuation policies, customers would be confused with that cloud(s) square measure appropriate for storing their knowledge and what hosting strategy is cheaper. the overall establishment is that customers sometimes place their knowledge into one cloud (which is subject to the seller lock-in risk) then merely trust to luck. supported comprehensive analysis of assorted progressive cloud vendors, this paper proposes a completely unique knowledge hosting theme (named CHARM) that integrates 2 key functions desired. the primary is choosing many appropriate clouds associate degreed an applicable redundancy strategy to store knowledge with reduced financial price and warranted handiness. The second is triggering a transition method to re-distribute knowledge per the variations of knowledge access pattern and valuation of clouds. we tend to valuate the performance of CHARM victimisation each trace-driven simulations and model experiments. The results show that compared with the main existing schemes, CHARM not solely saves around two hundredth of financial price however additionally exhibits sound ability to knowledge and worth changes.
\end{abstract}

Keywords: Enterprises, Enhance, Strategy, Integrates, Financial, Simulation.

\section{Existing System}

In existing industrial knowledge hosting systems, knowledge handiness (and reliability) square measure sometimes warranted by replication or erasure secret writing. within the multi-cloud state of affairs, we tend to additionally use them to fulfill totally different handiness necessities, however the implementation is totally different. For replication, replicas square measure place into many clouds, and a browse access is barely served (unless this cloud is unavailable then) by the "cheapest" cloud that charges negligible for out-going information measure and obtain operation. For erasure secret writing, knowledge is encoded into $\mathrm{n}$ blocks together with $\mathrm{m}$ knowledge blocks and $\mathrm{n} \square \mathrm{m}$ secret writing blocks, and these blocks square measure place into $\mathrm{n}$ totally different clouds. during this case, although knowledge handiness are often warranted with lower cupboard space(compared with

Received(February 13, 2017), Review Result(1st: February 28, 2017, 2nd: March 20, 2017), Accepted(April 10, 2017)

${ }^{1}$ Department of Computer Science and Engineering KL University, Vaddeswaram, Guntur psvssridhar@gmail.com

${ }^{2}$ Dept. of Multimedia, Hannam University, 70, Hannam-ro, Daedeok-gu, Daejeon, Korea, rdcaytiles@gmail.com 
replication), a browse access must be served by multiple clouds that store the corresponding knowledge blocks. Consequently, erasure secret writing cannot fill use of the most cost effective cloud as what replication will. Still worse, this defect are amplified within the multi-cloud state of affairs wherever information measure is usually (much) dearer than cupboard space.

\section{Proposed System:}

The planned CHARM theme. during this paper, we tend to propose a completely unique cost-effective knowledge hosting theme with high handiness in heterogenous multi-cloud, named "CHARM". It showing intelligence puts knowledge into multiple clouds with reduced financial price and warranted handiness. Specifically, we tend to mix the 2 wide used redundancy mechanisms, i.e., replication and erasure secret writing, into a consistent model to fulfill the desired handiness within the presence of various knowledge access patterns. Next, we tend to style associate degree economical heuristic-based algorithmic rule to settle on correct knowledge storage modes (involving each clouds and redundancy mechanisms). Moreover, we tend to implement the required procedure for storage mode transition (for with efficiency re-distributing data) by observance the variations of knowledge access patterns and valuation policies. we tend to valuate the performance of CHARM victimisation each tracedriven simulations and model experiments.The traces square measure collected from 2 on-line storage systems:, each of that possess many thousands of users. within the model experiments, we tend to replay samples from the 2 traces for a full month on high of 4 thought industrial clouds: Amazon S3, Windows Azure, Google Cloud Storage, and Aliyun OSS. analysis results show that compared with the main existing schemes which can be elaborate in $x$ VII-B), CHARM not solely saves around two hundredth (more well, seven-membered 44\%) of financial price however als

\section{Advantages:}

(1) Replication mechanism once the file's size is little. that's why grey level four puts its feet into the region of lower browse count and smaller file size. This storage mode table solely depends on costs of the obtainable clouds and needed

handiness. If the costs amendment, the table can amendment consequently, changing into a distinct one. 


\section{Drawback Statement}

(1) Nevertheless, as for multi-cloud individuals still encounter the 2 vital problems:

(1) the way to select applicable clouds to reduce financial price within the presence of heterogenous valuation policies?

(1) the way to meet the various handiness necessities of various services?

(1) As to financial price, [1]it primarily depends on the data-level usage, significantly storage capability consumption and network information measure consumption.

(1) As to handiness demand, the main concern lies within which redundancy mechanism (i.e., replication or erasure coding) is additional economical supported specific knowledge access patterns. In alternative words, here the basic challenge is:

(1) How to mix the 2 mechanisms elegantly therefore on greatly scale back financial price and in the meantime guarantee needed availability?

(1) Data Hosting and SMS square measure 2 necessary modules in CHARM. knowledge Hosting decides storage mode and therefore the clouds that the info ought to be hold on in.

(1) This may be a complicated whole number programming drawback incontestable within the following subsections. Then we tend to illustrate however SMS works well in $x \mathrm{~V}$, that is, once and the way again and again ought to the transition be enforced.

\section{Scope}

As a holistic storage system, there square measure many alternative factors to be thought of, like cache methods, geographical knowledge consistency, etc. However, we tend to solely target the info hosting strategy to reduce financial price whereas meeting versatile handiness necessities. although we've thought of the complexness and feasibleness once coming up with this strategy, the system style is out of the scope of this paper, and that we place the 
elaborated system style of multi-cloud knowledge hosting into future work. the complexness of this algorithmic rule is principally the primary loop, [2]and therefore the worst case complexness is $\mathrm{O}(\mathrm{Fn})$, wherever $\mathrm{Fn}$ is that the range of files. so as to cut back the complexness any, we are able to classify files with similar access patterns into teams, and implement transition within the unit of cluster. this can be out of the scope of this paper.

\section{Implementation of modules}

\section{Architecture:}

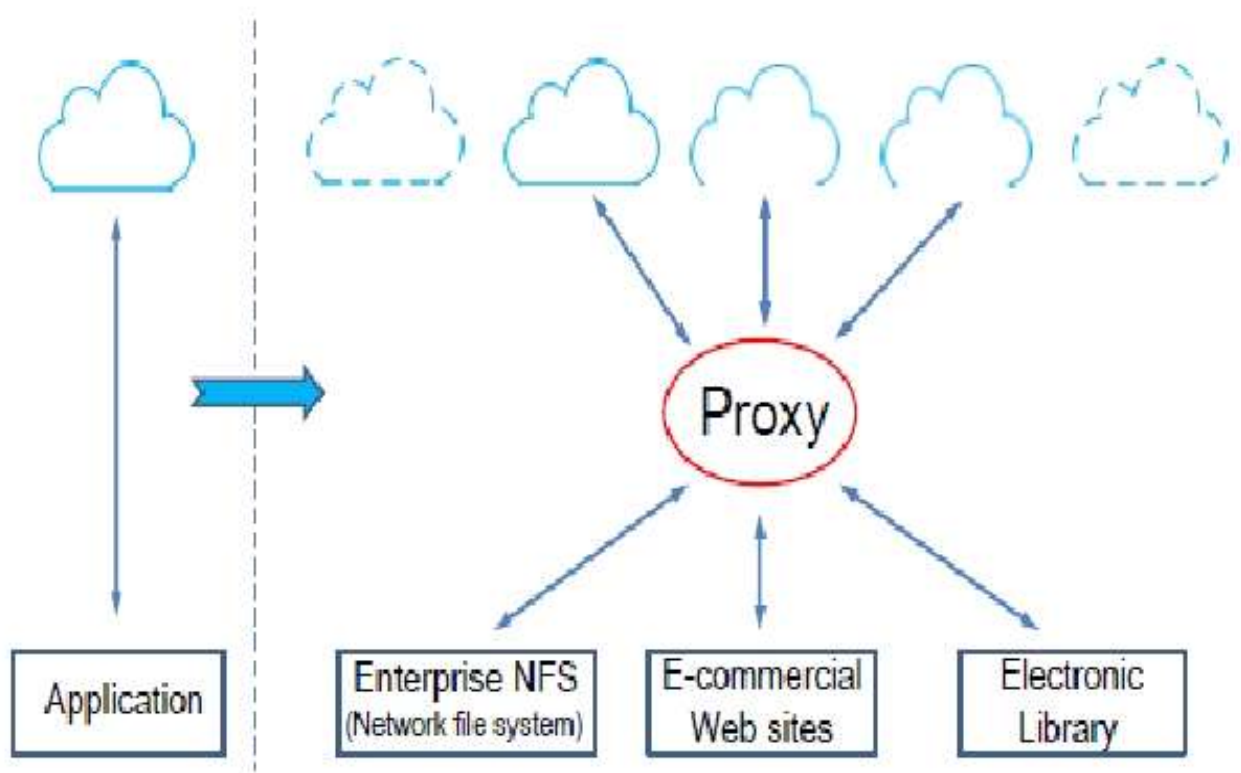




\section{Multi-cloud:}

Lots of information centers area unit distributed round the world, and one region like America, Asia, typically has many information centers happiness to a similar or totally different cloud suppliers. thus technically all the information centers is access by a user during a bound region, however the user would expertise totally different performance. The latency of some information centers is extremely low whereas that of some ones could also be intolerable high. CHARM chooses clouds for storing information from all the offered clouds that meet the performance demand, that is, they'll provide acceptable turnout and latency after they don't seem to be in outage. The storage mode transition doesn't impact the performance of the service. Since it's not a latency-sensitive method, we will decrease the priority of transition operations, and implement the transition in batch once the proxy has low employment.

\section{Information Hosting:}

In this section, we have a tendency to elaborate a efficient information hosting model with high accessibility in heterogenous multi-cloud, named "CHARM". The design of CHARM is shown in Figure three. the full model is found within the proxy in Figure one. There area unit four main parts in CHARM: information Hosting, Storage Mode shift (SMS), employment datum, and Predictor. employment datum keeps grouping and endeavor access logs to guide the position of knowledge. It conjointly sends datum data to Predictor that guides the action of SMS. information Hosting stores information mistreatment replication or erasure writing, in line with the scale and access frequency of the information. SMS decides whether or not the storage mode of bound information ought to be modified from replication to erasure writing or in reverse, in line with the output of Predictor. The implementation of fixing storage mode runs within the background, so as to not impact on-line service. Predictor is employed to predict the long run access frequency of files. The interval for prediction is one month, that is, we have a tendency to use the previous months to predict access frequency of files within the next month. [3] However, we have a tendency to don't place stress on the look of predictor, as a result of there are voluminous smart algorithms for prediction. Moreover, a awfullystraightforward predictor, that uses the weighted moving average approach, works well in our information hosting model. information Hosting and SMS area unit 2 necessary modules in CHARM. information Hosting decides storage mode and also the clouds that the information ought to be keep in. this can be a fancy whole number programming drawback incontestable 
within the following subsections. Then we have a tendency to illustrate however SMS works well in $x \mathrm{~V}$, that is, once and the way again and again ought to the transition be enforced.

\section{Cloud Storage:}

Cloud storage services became progressively in style. due to the importance of privacy, several cloud storage cryptography schemes are projected to safeguard information from people who don't have access. All such schemes assumed that cloud storage suppliers area unit safe and can't be hacked; but, in apply, some authorities (i.e., coercers) could force cloud storage suppliers to reveal user secrets or confidential information on the

cloud, therefore altogether circumventing storage cryptography schemes. during this paper, we have a tendency to gift our style for a replacement cloud storage cryptography theme that permits cloud storage suppliers to make convincing faux user secrets to safeguard user privacy. Since coercers cannot tell if obtained secrets area unit true or not, the cloud storage suppliers make sure that user privacy remains firmly protected. Most of the projected schemes assume cloud storage service suppliers or sure third parties handling key management area unit sure and can't be hacked; but, in apply, some entities could intercept communications between users and cloud storage suppliers and so compel storage suppliers to unleash user secrets by mistreatment government power or alternative means that. during this case, encrypted information area unit assumed to be illustrious and storage suppliers area unit requested to unleash user secrets. we have a tendency to aimed to create Associate in Nursing cryptography theme that might facilitate cloud storage suppliers avoid this difficulty. In our approach, we provide cloud storage suppliers means that to make faux user secrets. Given such faux user secrets, outside coercers will solely obtained cast information from a user's keep ciphertext. Once coercers suppose the received secrets area unit real, they're going to be glad and a lot of significantly cloud storage suppliers won't have unconcealed any real secrets. Therefore, user privacy remains protected. this idea comes from a special quite cryptography theme known as confutative cryptography.

\section{Owner Module:}

Owner module is to transfer their files mistreatment some access policy. initial they get the general public key for explicit transfer file when obtaining this public key owner request the key key for explicit transfer file. mistreatment that secret key owner transfer 
their file.

\section{User Module:}

This module is employed to assist the shopper to look the file mistreatment the file id and file name .[4]If the file id and name is wrong means that we have a tendency to don't get the file, otherwise server raise the general public key and find the cryptography file.If $\mathrm{u}$ wish the the cryptography file means that user have the key key.

\section{Algorithm:}

The key plan of this heuristic rule is delineate as follows: We initial assign every cloud a worth that is calculated supported four factors (i.e., accessibility, storage, bandwidth, and operation prices) to point the preference of a cloud. we elect the foremost most well-liked $n$ clouds, and so heuristically exchange the cloud within the most well-liked set with the cloud within the complementary set to look higher resolution. this can be kind of like the concept of Kernighan-Lin heuristic rule, that is applied to effectively partition graphs to attenuate the add of the prices on all edges cut. The preference of a cloud is wedged by the four factors, and that they have totally different weights. the provision is that the higher the higher, and also the worth is that the lower the higher. 


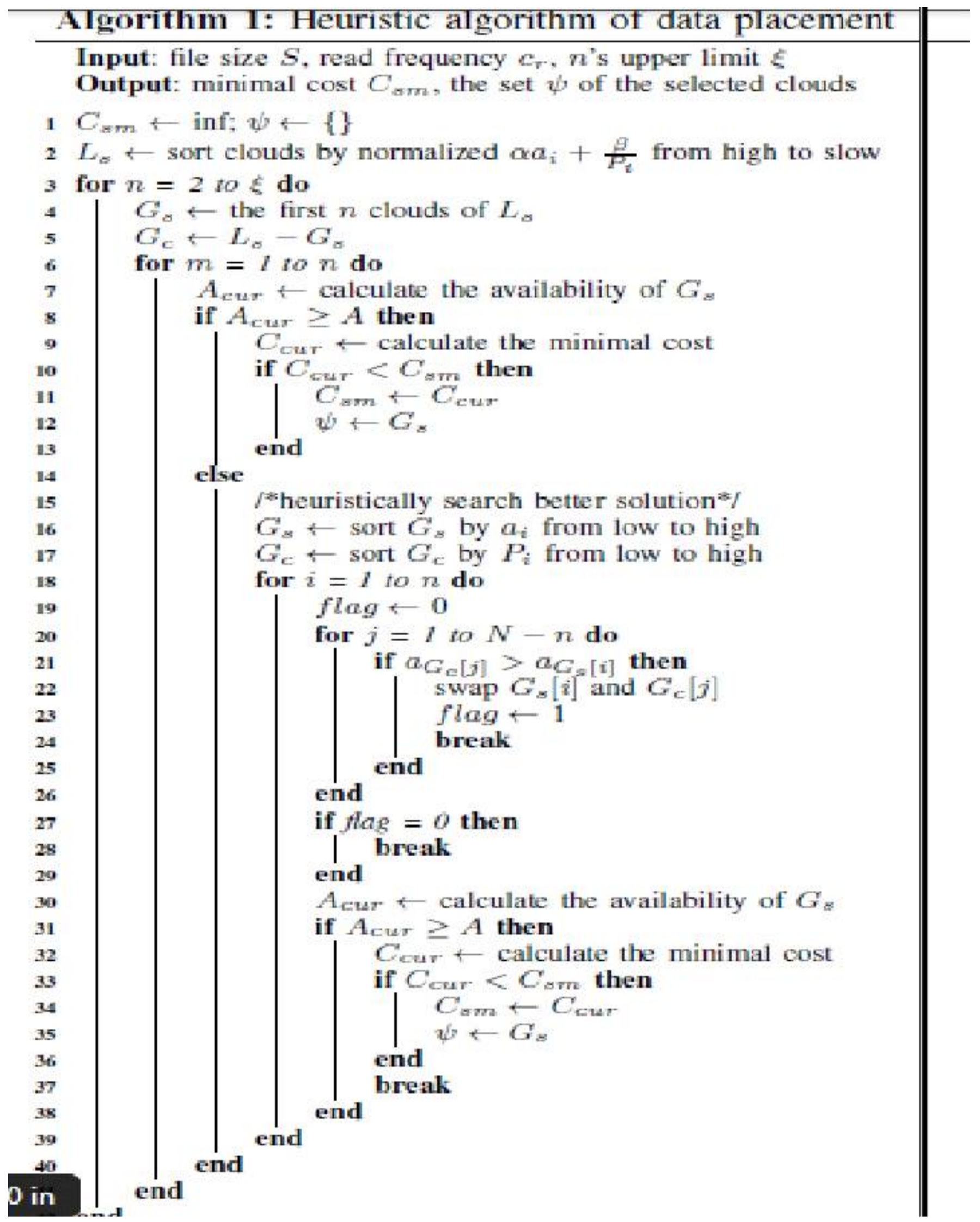




\section{Conclusion}

Cloud services are experiencing fast development conjointly the services supported multi-cloud also become prevailing. one in all the foremost issues, once moving services into clouds, is cost. So, during this paper, we have a tendency to style a completely unique storage theme CHARM, that guides customers to distribute information among clouds cost-effectively. CHARM makes fine-grained choices regarding that storage mode to use and that clouds to put information in. The analysis proves the potency of CHARM.

\section{Reference}

[1] M. Vukolic, The Byzantine empire in the intercloud, ACM SIGACT News, pp. 105-111, New York, September 2010.

[2] Sanjana Sharma, Swati Sengar, Sonika Soni, , Security in Cloud Computing, National Conference on Security Issues in Network Technologies, 2012.

[3] Bessani, M. Correia, B. Quaresma, F. Andre, and P. Sousa, "DepSky: Dependable and secure storage in a cloud-of-clouds," in Proc. 6th Conf. Comput.Syst., 2013

[4] H. Abu-Libdeh, L. Princehouse, H. Weatherspoon, "RACS: A case for cloud storage diversity", Proc. 1st ACM Symp. Cloud Comput., pp. 229-240, 2010. 